\section{Respostas cardiovasculares e avaliação da potência aeróbia em pessoas com lesão da medula espinhal}

\author{
Cardiovascular responses and assessment \\ of aerobic power in individuals with spinal \\ cord injury
}

Lucinar J. Forner Flores ${ }^{1}$

Melissa Antunes ${ }^{2}$

Anselmo Athayde Costa e Silva ${ }^{2}$

José Irineu Gorla ${ }^{3}$

\section{Resumo}

O objetivo deste estudo foi revisar na literatura atual os métodos e variáveis do consumo máximo de oxigênio/potência aeróbia e aspectos relacionados com a capacidade aeróbia em pessoas com lesão da medula espinhal. Foi utilizada a busca através da base de dados Sciverse - com os seguintes termos: Spinal cord injury, Tetraplegia, Paraplegia cruzados com Physical endurance, Exercise tolerance, Peak oxygen consumption. Para inclusão no estudo os artigos deveriam obedecer os seguintes critérios a) avaliação da potencia aeróbia em pessoas com lesão da medula espinhal na fase crônica da lesão, b) resultados com tetraplégicos ou paraplégicos, c) interação dos itens anteriores com $\mathrm{VO}_{2}$ máx $(1 / \mathrm{min}$ ou $\mathrm{ml} / \mathrm{kg} / \mathrm{min})$ e duração de esforço em cadeira de rodas, sendo publicados a partir do ano de 2000 até o presente momento da escrita do trabalho em 2012. Após cruzamentos de termos pré-selecionados, foram selecionados 26 artigos. A partir dos estudos revisados, observa-se uma grande variação de modelos, testes e maneiras de avaliar componentes fisiológicos relacionados aos sistemas cardiorrespiratórios e circulatórios. Esta gama de métodos parece estar relacionada à heterogeneidade da população/amostra e consequentemente, os resultados, por mais similares que sejam em alguns aspectos, apresentam valores discretos de proximidade, revelando uma necessidade de padronização para estes procedimentos nesta população. Com isto, é evidente a importância de estudos bem controlados e com nível tecnológico avançado para melhores prognósticos e acompanhamentos de pessoas com lesão da medula espinhal, seja para atividades esportivas, melhora de aspectos de riscos cardiovasculares ou para o cotidiano do dia a dia.

\section{Palavras-chave}

Consumo de oxigênio; Tetraplegia; Paraplegia.

\begin{abstract}
This study aimed to review in current literature methods and variables of maximal oxygen consumption, aerobic power and aspects related to aerobic capacity in people with spinal cord injury. The survey was through the Sciverse -website with the following terms: Spinal Cord Injury, quadriplegia, paraplegia crossed with physical endurance, exercise tolerance, peak oxygen power. To be included in the study the articles should present the following criteria a) assessment of aerobic power in people with spinal cord injury in the chronic phase of the injury, b) results with quadriplegics or paraplegics, c) interaction of the above items with $\mathrm{VO}_{2} \max (\mathrm{l} / \mathrm{min}$ or $\mathrm{ml} / \mathrm{kg} / \mathrm{min})$ and duration of effort in wheelchair, being published from year 2000 to date the year 2012. After crossing the pre-selected terms, 26 full articles were selected. From the reviewed studies, it was noticed a wide variation of models, tests and ways to evaluate physiological components related to cardiorespiratory and circulatory systems. This range of methods seems to be related to the heterogeneity of the population sample and therefore, even the results resembled in some aspects, they are discrete proximity values, revealing a necessity to standardize these procedures for this sample group. Studies well controlled with high technological level are important to establish better prognostics for people with spinal cord injuries, becoming possible to establish cardiovascular risk factors in sport practice or daily living activities in this population.
\end{abstract}

\section{Keywords}

Oxygen consumption; Tetraplegia; Paraplegia.
Rev Bras Ativ Fis Saúde p. 145-155 DOI:

http://dx.doi.org/10.12820/rbafs.v.18n2p145

${ }^{1}$ Curso de Educação Física, UNIOESTE, Santa Helena, PR, Brasil.

2 Faculdade de Educação Física, UNICAMP, Campinas, SP, Brasil.

3 Departamento de Estudos da Atividade Física Adaptada, Faculdade de Educação Física, UNICAMP, Campinas, SP, Brasil. 


\section{INTRODUÇÃO}

Atualmente tem crescido o número de mortes causadas por doenças relacionadas ao fenômeno hipocinesia. Doenças como diabetes, hipertensão e acidente vascular cerebral têm aumentado sua incidência ${ }^{1}$. Como uma forma de enfrentar este problema o treinamento físico regular é uma relevante opção, pois, confere efeitos benéficos diretamente relacionados aos domínios da aptidão física, como por exemplo, aspectos da composição corporal em que o exercício colabora para redução dos níveis de gordura corporal e aumento de massa magra ${ }^{2}$ e aspectos neuro-musculares em que ocorre aumento dos níveis de força que podem estar relacionados a aumentos da funcionalidade.

Com relação aos aspectos cardiovasculares as melhoras decorrentes do exercício ocorrem, em parte, por que o treinamento físico melhora a capacidade de trabalho do músculo esquelético, aumentando a condutância na circulação periférica; além disso, aprimora também a modulação extrínseca do coração e intrínseca da bomba cardíaca ${ }^{2}$. Evidências sugerem que a magnitude desses benefícios citados aumenta proporcionalmente com a intensidade das sessões de exercício físico que constituem o programa de treinamento físico. Gulatti $e \mathrm{al}^{3}$ sugerem que os efeitos benéficos do exercício físico regular podem depender da intensidade e volume de trabalho realizado.

A potência aeróbia máxima, definida como a máxima absorção, transporte e consumo de oxigênio $\left(\mathrm{VO}_{2 \max }\right)$ é, geralmente, considerado o melhor marcador para a capacidade funcional do sistema cardiorrespiratório. A medida direta de $\mathrm{VO}_{2 \text { máx }}$ durante um teste máximo ergométrico é considerada o melhor método para avaliar a potência aeróbia ${ }^{4}$.

A lesão da medula espinhal (LME) provoca alterações motoras e sensoriais no indivíduo, acarretando consequências sociais na vida da maioria dessas pessoas, especialmente para aqueles com lesão alta (lesão acima da $1^{\text {a }}$ vértebra torácica) e, consequentemente, capacidade física reduzida. Essa capacidade pode ser definida por uma série de componentes inter-relacionados, tais como, $\mathrm{VO}_{2}$ máx, força muscular e função cardiovascular e pulmonar ${ }^{1}$. Além da disfunção física e sensorial, a LME provoca várias sequelas, entre elas, a redução da capacidade cardiorrespiratória, consequência direta da mobilidade reduzida em decorrência da paralisia ${ }^{5}$. A vida dependente da cadeira de rodas implica na dificuldade em manter um estilo de vida ativo, o que, juntamente com um estado sedentário, promove o desenvolvimento de quadros de obesidade, síndrome metabólica, diabetes e doenças cardiovasculares que limitam as repostas fisiológicas à atividade motora, e conduzem à rápida instalação da fadiga ${ }^{1}$.

A redução do nível de atividade física e as alterações adversas na composição corporal causadas pela lesão têm consequências metabólicas que podem influenciar o avanço e a gravidade de doenças cardiovasculares ${ }^{6}$. Com isto, é evidente a importância de estudos bem controlados e com nível tecnológico avançado para melhores prognósticos e acompanhamentos de pessoas com lesão da medula espinhal, seja para atividades esportivas, melhora de aspectos de riscos cardiovasculares ou para o cotidiano do dia a dia.

Stevens et al. ${ }^{7}$ demonstraram que existe uma importante correlação positiva entre a qualidade de vida e nível da prática de atividade física em indivíduos com LME. Sendo assim, o engajamento desses indivíduos em programas regulares de exercícios físicos deve ser incentivado, pois, trata-se de uma abordagem promissora, com boa proporção custo-benefício, e eficaz na promoção da saúde e 
da qualidade de vida, trazendo inúmeros benefícios que se revelam na melhora do desempenho da vida diária, na promoção do bem estar físico e social e na redução da incidência de complicações clínicas, favorecendo a independência funcional desses indivíduos.

O objetivo do presente estudo foi revisar na literatura atual os métodos e variáveis para avaliação do consumo máximo de oxigênio/potência aeróbia e aspectos relacionados com a capacidade física aeróbia em pessoas com lesão da medula espinhal devido a importância desta variável para o desenvolvimentos de programas de exercício físico na população específica pessoas com LME.

\section{MÉTODOS}

Este trabalho caracterizou-se como uma revisão sistemática de literatura que consiste em análise da produção bibliográfica em determinada área temática, dentro de um recorte de tempo, e fornece uma visão geral do problema pesquisado, evidenciando novas ideias, métodos e sub-temas ${ }^{8}$. Foi utilizada a busca através do site de busca integrada Sciverse (www.hub.sciverse.com) o qual integra as bases PUBMED, Sciense Direct, Scopus entre outras. Os unitermos utilizados foram: Spinal Cord Injury, tetraplegia, paraplegia cruzados com physical endurance, exercise tolerance, peak oxygen consumption. Para inclusão no estudo os artigos deveriam obedecer os seguintes critérios a) avaliação da potencia aeróbia em pessoas com lesão da medula espinhal na fase crônica da lesão, b) resultados com tetraplégicos ou paraplégicos, c) interação dos itens anteriores com $\mathrm{VO}_{2 \max }(1 / \mathrm{min}$ ou $\mathrm{ml} / \mathrm{kg} /$ min) e duração de esforço em cadeira de rodas, sendo publicados a partir do ano de 2000 até o presente momento do ano de 2012.

A base estrutural adotada seguiu o formato aplicado por Haisma et al. ${ }^{9}$. Foram encontrados 296 artigos com os termos usados para a pesquisa inicial nas bases de dados. Após cruzamentos de termos pré-selecionados, foram selecionados 26 artigos completos, sendo 3 revisões e os demais artigos originais de revistas nacionais e internacionais, sendo que foram excluídos artigos que não lidavam com sujeitos atletas de esporte em cadeira de rodas.

\section{RESULTADOS}

$\mathrm{Na}$ revisão de Theisen e Vanlandewijck ${ }^{10}$, encontrou-se evidencia de que os resultados cardiovasculares apresentados em relação ao volume sistólico menor nas pessoas com lesão da medula espinhal (paraplégicos), quando comparados com pessoas ditas "saudáveis" do grupo controle (sem lesão de medula), e uma FC maior para valores de $\mathrm{VO}_{2}$ submáximo para o grupo LME quando em comparação ao grupo controle. Este fator é relacionado à redução da atividade simpática, ocorrida após lesão medular. A FC máxima em exercícios normalmente é bastante afetada principalmente em lesados acima da $6^{\mathrm{a}}$ vértebra torácica (T6), ficando o relato de valores entre 115 - $130 \mathrm{bpm}$, conforme estes mesmo autores.

Outra importante revisão realizada dentro do período proposto deste estudo foi a de Teasell e colaboradores ${ }^{11}$, na qual os autores evidenciam a discussão das consequências cardiovasculares após lesão medular e controle do sistema nervoso simpático. Num destes textos citados, Eriksson e colaboradores (1988) apud Teassel et al. ${ }^{11}$ avaliaram 58 homens (lesões medulares entre C4 e L4) sendo 25 treinados, 33 não treinados. Durante o exercício físico máximo em cadeira de rodas, a média de FC apresentada foi de $119 \mathrm{bpm}$ para não treinados e $118 \mathrm{bpm}$ 
para os treinados, não apresentando diferenças estatísticas na média de FC em teste máximo entre pessoas com lesão da medula espinhal pareados por status de treinamento, o que leva a crer que o exercício físico regular não conseguiu auxiliar a atuação da atividade simpática cardíaca nesta amostra ${ }^{12}$.

Lovell et al. ${ }^{13}$ realizaram uma investigação cuja proposta era comparar as respostas cardiorrespiratórias e eficiência mecânica de atletas de ciclismo com lesão da medula espinhal $(n=10)$ com sujeitos não atletas com LME $(n=10)$. Para tanto os participantes foram submetidos a um teste aeróbio sub-máximo em ergômetro de braço, com dois estágios constantes de 4 minutos separados por um minuto de intervalo. $\mathrm{O}$ grupo atletas apresentou $\mathrm{VO}_{2}$ pico de $40,4 \pm 5,5 \mathrm{ml} / \mathrm{kg} / \mathrm{min}$ significativamente maior do que o outro de sujeitos não atletas $(21,23 \pm 4,7 \mathrm{ml} / \mathrm{kg} / \mathrm{min})$.

Bernard et al. ${ }^{14}$, avaliaram 12 indivíduos (média de idade 30 anos) atletas com paraplegia, divididos em dois grupos, um com lesão de nível alto (acima da oitava vértebra torácica) e outro baixo. A avaliação cardiorrespiratória ocorreu na cadeira de rodas em esteira rolante com incremento de carga de $1 \mathrm{~km} / \mathrm{h} / \mathrm{min}$, iniciando o teste com $4 \mathrm{~km} / \mathrm{h}$. Durante o exercício máximo, não foram observadas diferenças significativas entre os dois grupos para os valores cardiorrespiratórios e ventilatórios. Apesar da ausência de diferenças significativas, os maiores valores máximos alcançados foram do grupo com lesão baixa, confirmando a maior capacidade de adaptação ao exercício físico no grupo de lesão torácica inferior. Também com paraplégicos Knechtle e Kopfli. ${ }^{15}$, analisaram 11 jogadores de basquete em cadeira de rodas (média de idade de 28 anos). Neste estudo, foi aplicado um protocolo de exercício físico com aumento da inclinação na esteira para verificação da possibilidade de utilização de um protocolo com inclinação na esteira em pessoas com lesão da medula espinhal (abaixo de T1). Oito jogadores tinham LME, dois tinham lesão do sistema nervoso central e um teve poliomielite. Foram mensurados o consumo máximo de oxigênio $\left(\mathrm{VO}_{2}\right.$ máx) e a frequência cardíaca $(\mathrm{FC})$, enquanto o nível de lactato foi determinado imediatamente após o protocolo de exercício. Os atletas apresentaram aumentos significativos em relação à frequência cardíaca máxima $(185 \pm 11,4 \mathrm{bpm})$, lactato máximo $(10,2 \pm 2,1 \mathrm{mmol} / 1)$ e $\mathrm{VO}_{2}$ máx $(35,1 \pm 4,9$ $\mathrm{ml} / \mathrm{min} / \mathrm{kg}$ ). Diante dos resultados, ficou constatado que um protocolo de exercício com inclinação crescente é uma alternativa válida de avaliação do componente aeróbio em comparação com um protocolo de exercício com velocidade crescente.

Mantendo a sequência de análises com sujeitos paraplégicos Jacobs, Nash e Rusinowski ${ }^{16}$ avaliaram os efeitos do treinamento em circuito sobre a capacidade cardiorrespiratória e força muscular. Dez homens com paraplegia nos níveis entre T5-L1 participaram do estudo. Os indivíduos completaram 12 semanas de treinamento em circuito, usando uma série de exercícios alternados de resistência muscular e ergometria de braço de baixa resistência. Após o protocolo de treinamento, foram testadas a força isocinética da extremidade superior e a capacidade cardiorrespiratória. Aumentos significativos foram observados no consumo máximo de oxigênio $(29,7 \%, p<0,01)$ e na potência de pico durante o teste do braço $(p<0,05)$. Este modelo de treinamento se mostrou eficaz para melhora cardiovascular e na capacidade de força dos participantes do programa de exercícios físicos regulares.

Outro exemplo importante, da aplicação do treinamento físico como situação de intervenção em pessoas com lesão da medula espinhal foi o de Bougenot et al., ${ }^{17}$ no qual participaram sete indivíduos paraplégicos não treinados do sexo masculino com média de 35 anos de idade e que apresentavam pelo menos 12 anos pós lesão. Foram avaliados os efeitos de um programa de treinamento com duração de 6 semanas, sobre variáveis cardiorrespiratórias em teste ergométrico com incre- 
mento de carga de $10 \mathrm{~W}$ a cada dois minutos e iniciando o teste com $15 \mathrm{~W}$. O protocolo de treinamento compunha-se de 45 minutos propulsão em esteira rolante, três vezes por semana. Os resultados obtidos mostraram aumentos significativos das variáveis $\mathrm{VO}_{2}$ máx e potência pico (watts). $\mathrm{O}$ treinamento físico realizado em ergômetro de braço em pessoas com lesão da medula espinhal foi eficiente na melhora de variáveis cardiovasculares, concluindo que o treinamento físico traz adaptações positivas para a aptidão física e capacidade aeróbia.

Vanderthommen et al. ${ }^{18}$ avaliaram 37 indivíduos (sendo: 26 paraplégicos, dois tetraplégicos, cinco com sequelas de poliomielite e quatro amputados; os autores relataram a participação de duas mulheres, porém não descriminaram o tipo de deficiência) através de proposta de teste progressivo de quadra (octogonal) para usuários de cadeira de rodas, no qual foi usado protocolo intermitente/progressivo com bip sonoro. Os valores médios do grupo estudado foram de: idade 36,5 anos; massa corporal 77,7kg; estatura $177 \mathrm{~cm}$; tempo de lesão 16,5 anos e tempo de uso da cadeira de rodas 11 anos. $\mathrm{O}$ registro do $\mathrm{VO}_{2 \text { máx }}$ foi através da análise direta de gases no equipamento portátil (K4b2 system). Já a análise da concentração de lactato sanguíneo foi realizada através YSI modelo 1500 (Yellow springs instruments). Os resultados encontrados no estudo foram: $25,2 \pm 5,9 \mathrm{ml} / \mathrm{kg} / \mathrm{min}$ de $\mathrm{VO}_{2}$ máx/ pico; $172 \pm 26 \mathrm{bpm}$ de FC máx; $5,4 \pm 1,9 \mathrm{mmol} / 1$ de lactato máximo. Diante dos resultados, a proposta de teste progressivo foi considerada válida para avaliação aptidão física em usuários de cadeira de rodas.

Com uma amostra expressiva para as características dos estudos na área da atividade física adaptada Janssen e colaboradores ${ }^{19}$ avaliaram 166 indivíduos (20 mulheres). No entanto foi observada grande heterogeneidade da amostra com relação as variáveis idade, massa corporal, nível da lesão, tempo de lesão, e o nível de realização de atividades físicas. Todos os participantes da pesquisa apresentavam lesão por, pelo menos, 7 anos e eram praticantes de atividades físicas regulares por, no mínimo, $4 \mathrm{~h}$ semanais. $\mathrm{O}$ estudo consistiu em um teste progressivo, em cadeira de rodas, para determinar o consumo máximo de oxigênio e a potência máxima, incluindo também um teste de sprint em cadeira de rodas para determinar a potência anaeróbia e um teste de força isométrica. Embora a variação de resultados tenha sido entre 48 a $80 \%$, o que pode ser explicada pela heterogeneidade da amostra (nível da lesão, o nível de atividade, sexo, idade, massa corporal, e tempo de lesão), os pesquisadores concluíram que, ainda que a capacidade física seja amplamente determinada por fatores que não podem ser alterados na população com LME - como o nível da lesão, idade e sexo - os fatores mutáveis, como o nível de atividade física habitual e a composição corporal (baixo componente de gordura), desempenharam um papel adicional nesta amostra para determinação dos melhores resultados ${ }^{19}$.

Dallmeijer et al. ${ }^{20}$ compararam respostas cardiorrespiratórias em um modelo de ergômetro de braço e em cadeira de rodas. Participaram do estudo 2 grupos subdivididos em nove indivíduos com lesão medular (paraplégicos) e dez sem lesão medular do sexo masculino (36 anos). Duas séries de 4 minutos de exercício físico submáximo em 25 e 35 Watts de potência, seguidos por exercício de 1 minuto com potência crescente até a exaustão, foi o protocolo utilizado. Os resultados mostraram menores valores de $\mathrm{VO}_{2 \max }$, ventilaçãa (VE), FC, taxa de percepção de esforço e uma maior eficiência bruta no ciclo ergômetro de braço em ambos os grupos, enquanto que não foram encontradas diferenças significativas em potência pico e pico de $\mathrm{VO}_{2}$, VE e $\mathrm{FC}$ quando usado o ciclo ergômetro de braço em ambos os grupos. Para este estudo, ficou evidenciado a igualdade de respostas cardior- 
respiratórias em ambos os grupos, mesmo utilizando-se de diferentes modelos de equipamentos para a avaliação aeróbia. Estes resultados ainda indicam a possibilidade de usuários de cadeira de rodas usarem o modelo de ergômetro de braço, para as atividades diárias, pois possui uma maior mobilidade e não prejudicou significativamente as respostas cardiovasculares e respiratórias analisadas.

Em uma interessante comparação entre um grupo de indivíduos com diferentes níveis de lesão e outro grupo controle sem LME, Hopman et al. ${ }^{21}$ avaliaram, em ciclo ergômetro de braço com incremento de carga de três, cinco ou $10 \mathrm{~W} / \mathrm{min}$, seis indivíduos paraplégicos e seis indivíduos tetraplégicos com média de idade de 33 anos (10 sujeitos no grupo controle), praticantes de atividade física regular por pelos menos $4 \mathrm{~h} /$ semana. $\mathrm{O}$ consumo de oxigênio durante o exercício aumentou no grupo controle sem deficiência, bem como em pessoas com paraplegia e, em menor grau, nas pessoas com tetraplegia, indicando que o consumo máximo de oxigênio durante o exercício físico de braço é limitado pelo fornecimento de oxigênio ao invés do tamanho da massa muscular envolvida e pequenas limitações bioquímicas relacionadas.

Knechtle et al. ${ }^{22}$ avaliaram 10 atletas usuários de cadeiras de rodas treinados e 10 ciclistas treinados com o objetivo de determinar a taxa de oxidação de gordura, a fim de fornecer recomendações para a utilização de ciclo ergômetro. Foi avaliada a capacidade aeróbia através do teste de $\mathrm{VO}_{2 \operatorname{máx}}$ em um ciclo ergômetro de braço e bicicleta ergométrica, respectivamente. Os atletas deficientes mostraram uma tendência de maior concentração de lactato em cada intensidade em relação aos ciclistas. Os atletas usuários de cadeiras de rodas bem treinados alcançaram a maior oxidação de gordura no ciclo ergômetro. Os resultados sugerem que os atletas em cadeiras de rodas bem treinados podem executar o treinamento em ciclo ergômetro, criando assim novas possibilidades de treinamento.

Em uma amostra brasileira ${ }^{5}$, as análises cardiorrespiratórias com paraplégicos $(n=5)$ e tetraplégicos $(n=5)$, foram realizadas em repouso, durante estimulação elétrica neuro-muscular (EENM) de 20 minutos do quadríceps direito/esquerdo e durante a recuperação deste estímulo. $O$ grupo paraplégico apresentou média de massa corporal de $84,6 \pm 14,1 \mathrm{~kg}$ e estatura de $177 \pm 7,8 \mathrm{~cm}$. Já no grupo tetraplégico, os valores foram de $62,4 \pm 5,02 \mathrm{~kg}$ e $177,2 \pm 6,94 \mathrm{~cm}$. A pressão arterial foi mensurada via método auscultatório. A FC foi monitorada por eletrocardiograma e a avaliação metabólica cardiorrespiratória foi avaliada por analisador de gases Sensormedics V29C. Os valores dos grupos paraplégicos e tetraplégicos são apresentados nas tabelas a seguir:

Tabela 1 - Valores cardiorrespiratórios de para e tetraplégicos.

\begin{tabular}{lcccccc}
\hline & \multicolumn{2}{c}{ Repouso } & \multicolumn{2}{c}{ EENM } & \multicolumn{2}{c}{ Recuperação } \\
\hline & Para & tetra & para & Tetra & Para & Tetra \\
\hline $\mathrm{VO}_{2}$ & $0,22 \pm 0,02$ & $0,15 \pm 0,01$ & $0,48 \pm 0,01$ & $0,29 \pm 0,02$ & $0,25 \pm 0,02$ & $0,16 \pm 0,01$ \\
\hline $\mathrm{VCO}_{2}$ & $0,15 \pm 0,01$ & $0,10 \pm 0,01$ & $0,4 \pm 0,08$ & $0,23 \pm 0,02$ & $0,19 \pm 0,02$ & $0,12 \pm 0,01$ \\
$\mathrm{FC}$ & $85,4 \pm 8,01$ & $67,39 \pm 5,65$ & $99,25 \pm 9,57$ & $89,64 \pm 8,22$ & $93,99 \pm 7,65$ & $83,84 \pm 6,28$ \\
PA Sistólica & $116 \pm 5,4$ & $104 \pm 4,41$ & $144 \pm 5,4$ & $128 \pm 5,03$ & $121 \pm 8,94$ & $112 \pm 4,47$ \\
PA Diastólica & $76 \pm 5,4$ & $68 \pm 3,36$ & $74 \pm 8,94$ & $70 \pm 6,36$ & $74 \pm 8,94$ & $70 \pm 5,67$ \\
\hline
\end{tabular}

$\mathrm{VO}_{2}$ - Consumo máximo de oxigênio em litros/minuto ( $\left./ / \mathrm{min}\right) ; \mathrm{VCO}_{2}$ - Produção de dióxido de carbono em litros/minuto (I/min); FC - Frequência Cardíaca em batimentos por minuto (bpm); PA Sistólica - Pressão arterial sistólica em milímetros de mercúrio $(\mathrm{mmHg})$; PA Diastólica - Pressão arterial diastólica em milímetros de mercúrio ( $\mathrm{mmHg}$ ); Adaptado de Paolillo, Paolillo e Cliquet Jr., (2005). 
Foram encontrados resultados, no estudo supracitado, de consumo de oxigênio inversamente proporcionais a altura da lesão medular, pois, observou-se uma relação inversamente proporcional entre nível da lesão e valores de consumo de oxigênio, ou seja, quanto mais alta a lesão (tetraplegia), menor os valores de $\mathrm{VO}_{2 \text { máx. }}$ Também foi observada bradicardia e hipotensão no grupo tetraplégico durante o repouso. Durante a estimulação, ocorreram aumentos da FC e pressão arterial sistólica, demonstrando atuação mais ativa do tônus simpático nestas pessoas com lesão da medula espinhal. Os autores ressaltaram a capacidade dos avaliados em conseguirem respostas dentro da normalidade durante o exercício físico.

Hayes e colaboradores ${ }^{23}$ realizaram um teste incremental de ergômetro de braço. Para isto, 13 sujeitos com lesão da medula espinhal (uma mulher, três tetraplégicos) com idade variando de 30 a 72 anos foram avaliados em um ergômetro de braço da marca Monark ${ }^{\circledR}$ com analisador de gases (K4b system). A média de FC máx durante o teste foi de 143 bpm, com amplitude de valores de 96 a 216 bpm. Já o $\mathrm{VO}_{2}$ pico teve média de $16,36 \mathrm{ml} / \mathrm{kg} / \mathrm{min}$ e variou de 10,66 a $27,17 \mathrm{ml} / \mathrm{kg} / \mathrm{min}$. Estes valores foram semelhantes aos de resultados encontrados em outros estudos, mas a amplitude dos resultados demonstrou a não homogeneidade da amostra estudada, o que dificulta determinar parâmetros de variáveis aeróbias em pessoas com lesão da medula espinhal.

Ainda com a utilização da avaliação direta do consumo de $\mathrm{O}_{2}$ e produção de $\mathrm{CO}_{2}$, sete indivíduos com LME (paraplégicos) foram avaliados para determinar o impacto da ergonomia e variações do ambiente/cadeira/cambagens de rodas em testes de quadra. Foram avaliados variáveis de componentes cardiorrespiratórios através do analisador de gases (K4b2 system). Os resultados apresentaram valores de mediana do $\mathrm{VO}_{2 \text { pico }} 29,07 \mathrm{ml} / \mathrm{kg} / \mathrm{min}-\mathrm{VCO}_{2}$ de $35,33 \mathrm{ml} / \mathrm{kg} / \mathrm{min}-\mathrm{FC}_{\text {máx }}$ de $181 \mathrm{bpm}{ }^{24}$.

Goosey-Tolfrey e Tolfrey ${ }^{25}$ avaliaram 24 jogadores de basquete em cadeira de rodas. $\mathrm{O}$ protocolo consistiu na utilização de espaço de 20 metros em quadra com velocidade inicial de $2,36 \mathrm{~m} / \mathrm{s}$ e incrementos a cada minuto de $0,14 \mathrm{~m} / \mathrm{s}$ até a exaustão. Para verificação de correlação, foi utilizado também o protocolo de ergômetro computadorizado em cadeira de rodas ${ }^{26}$. A análise do $\mathrm{VO}_{2}$ foi através de medida direta (Havard Apparatus $^{\odot}$ ) e a FC através de frequencímetro (Polar ${ }^{\odot}$ ). Os resultados apresentados foram: idade $29 \pm 6$ anos; massa corporal 73,7 $\pm 10.9 \mathrm{~kg}$; $\mathrm{VO}_{2 \text { pico }}$ por ergoespirometria direta 2,66 $\pm 0,491 / \mathrm{min} ; \mathrm{FC}$ máx na ergoespirometria direta $188 \pm 10 \mathrm{bpm}$; Distância percorrida no teste de quadra $2056 \pm 272$ metros e FC máx no teste de quadra: $186 \pm 11 \mathrm{bpm}$. Em recente estudo deste mesmo grupo, Leicht, Bishop e Goosey-Tolfrey ${ }^{27}$ verificaram respostas relacionadas ao exercício submáximo ( $40 \%$ a $80 \% \mathrm{VO}_{2}$ pico) em atletas usuários de cadeiras de rodas, tetraplégicos, paraplégicos e não lesados. Os tetraplégicos avaliados pertenciam a uma equipe de Rugby em cadeira de rodas ( $\mathrm{n}=8$ ) com experiência de 7,8 anos no esporte. $\mathrm{O} \mathrm{VO}_{2}$ pico foi de 24,5 + 4,9 ml/kg/min, com FC máxima de 129-12 bpm e pico de lactato de 4,95-1,28 mmol. O grupo paraplégico avaliado participa do basquetebol em cadeira de rodas com experiência de 12,2 + 5,3 anos. Este grupo apresentou valores significativamente maiores que o grupo tetraplégico nas variáveis. $\mathrm{O} \mathrm{VO}_{2}$ pico foi de $34,9 \pm 5,1 \mathrm{ml} / \mathrm{kg} / \mathrm{min}$, com FC máxima de $184 \pm 10 \mathrm{bpm}$ e pico de lactato de $8,47 \pm 2,75 \mathrm{mmol}$. Estes números também indicam que a altura da lesão (quanto mais alta a lesão menor a capacidade aeróbia e suas variáveis) influencia nos resultados do componente aeróbio.

Morgulec-Andamowicz et al. ${ }^{28}$ avaliaram 30 atletas de Rugby em Cadeira de Rodas - RCR da Polônia, os quais foram divididos em 4 grupos conforme classi- 
ficação funcional do RCR. O objetivo era verificar a existência de diferenças entre os grupos para variáveis aeróbias, anaeróbias e de habilidades no RCR. Foram realizados testes através de exercício aeróbio máximo em esteira para cadeira de rodas com incrementos até a exaustão e Wingate test em ergômetro de braço para avaliação da potência. Ao contrário do estudo anterior ${ }^{24}$ os resultados apresentados foram: $\mathrm{VO}_{\text {2pico }} 21,1 \pm 6,3$ (grupo I menor classificação funcional) a 30,2 $\pm 7,2$ $\mathrm{ml} / \mathrm{kg} / \mathrm{min}$ (grupo IV maior classificação funcional); Potência Pico $83 \pm 35$ (grupo I menor classificação funcional) a $226 \pm 28 \mathrm{~W}$ (grupo IV maior classificação funcional). Diante destes resultados os autores afirmaram que a classificação funcional não interferiu nas respostas das variáveis avaliadas (principalmente na potência aeróbia), pois as diferenças entre os grupos só foram significativas entre o grupo I e grupo IV e não ocorreram para os grupos intermediários do RCR.

No Quadro 01 são sintetizados os principais aspectos relacionados à potência aeróbia nos artigos aqui apresentados para uma melhor visualização e obtenção das informações inerentes a cada estudo.

\begin{tabular}{|c|c|c|c|c|}
\hline Autores/ano & Objetivos & Amostra & Materiais e Métodos & Resultados/ Considerações \\
\hline $\begin{array}{l}\text { Bernard et al., } \\
(2000)\end{array}$ & $\begin{array}{l}\text { Comparar aspectos de } \\
\text { potência aeróbia entre } \\
\text { grupo com lesão baixa } \\
\text { e lesão alta; }\end{array}$ & $\begin{array}{l}12 \text { atletas- } \\
\text { paraplégicos } \\
\text { (alto e baixo); }\end{array}$ & $\begin{array}{l}\text { Cadeira de rodas em esteira- } \\
\text { teste máximo- incremento } \\
\text { de } 1 \mathrm{~km} / \mathrm{h} / \mathrm{min} \text {; }\end{array}$ & $\begin{array}{l}\text { Sem diferenças entre os grupos. } \\
\text { Maiores valores no grupo lesão baixa. }\end{array}$ \\
\hline $\begin{array}{l}\text { Knechtle e Kopfli } \\
\text { (2001) }\end{array}$ & $\begin{array}{l}\text { Verificar a } \\
\text { possibilidade de } \\
\text { uso de teste com } \\
\text { inclinação em usuários } \\
\text { de cadeira de rodas; }\end{array}$ & $\begin{array}{l}11 \text { jogadores } \\
\text { de basquete em } \\
\text { cadeira de rodas- } 8 \\
\text { LME- torácica }\end{array}$ & $\begin{array}{l}\text { Cadeira de rodas em } \\
\text { esteira- com inclinação-teste } \\
\text { máximo; Níveis de lactato e } \\
\text { FC máx. }\end{array}$ & $\begin{array}{l}\text { Valores médios de } \mathrm{VO}_{2} \text { máx } \\
35,1 \pm 4,9 \mathrm{ml} / \mathrm{kg} / \mathrm{min} \text { - lactato de } 10,2 \pm 2,1 \\
\text { mmol e } \mathrm{FC} \text { Max de } 185 \pm 11,4 \mathrm{bpm} \text {. Foi } \\
\text { constatado que o teste com inclinação é } \\
\text { algo válido na avaliação de usuários de } \\
\text { cadeira de rodas. }\end{array}$ \\
\hline $\begin{array}{l}\text { Jacobs, Nash e } \\
\text { Rusinowski (2001) }\end{array}$ & $\begin{array}{l}\text { Avaliar efeitos } \\
\text { do treinamento } \\
\text { em circuito em } \\
\text { paraplégicos; }\end{array}$ & $\begin{array}{l}10 \text { homens } \\
\text { paraplégicos; }\end{array}$ & $\begin{array}{l}12 \text { semanas de treino em } \\
\text { circuito- Exercícios resistidos } \\
\text { e ergometria de braço com } \\
\text { baixa intensidade; }\end{array}$ & $\begin{array}{l}\text { Aumentos significativos ( } 29,7 \%) \text { no } \\
\mathrm{VO}_{2} \text { máx e na potência pico durante } \\
\text { o teste com os braços. Este modelo } \\
\text { de treino foi eficaz para melhora } \\
\text { cardiovascular e capacidade de força. }\end{array}$ \\
\hline $\begin{array}{l}\text { Bougenot et al., } \\
\text { (2003) }\end{array}$ & $\begin{array}{l}\text { Avaliar efeitos do } \\
\text { treinamento de } 6 \\
\text { semanas nas variáveis } \\
\text { cardiorrespiratória; }\end{array}$ & $\begin{array}{l}7 \text { paraplégicos não } \\
\text { treinados- homens- } \\
\text { lesão com } 12 \text { anos e } \\
35 \text { anos de idade; }\end{array}$ & $\begin{array}{l}\text { Treino de } 45 \text { min de } \\
\text { ergômetro de braço } 3 x \text { por } \\
\text { semana; }\end{array}$ & $\begin{array}{l}\text { Aumentos significativos da potência } \\
\text { pico (watts) e } \mathrm{VO}_{2} \text { máx. O treinamento } \\
\text { de } 6 \text { semanas foi eficiente nas variáveis } \\
\text { avaliadas. }\end{array}$ \\
\hline $\begin{array}{l}\text { Vanderthommen et } \\
\text { al., (2002) }\end{array}$ & $\begin{array}{l}\text { Propor teste } \\
\text { progressivo em } \\
\text { quadra; }\end{array}$ & $\begin{array}{l}37 \text { paraplégicos ( } 2 \\
\text { mulheres)- média } \\
\text { de idade } 36,5 \text { anos; }\end{array}$ & $\begin{array}{l}\mathrm{VO}_{2} \text { máx direto-através do } \\
\mathrm{K} 4 \text { portátil; Fc máx e lactato; }\end{array}$ & $\begin{array}{l}\text { Teste considerado apto para usuários de } \\
\text { cadeira de rodas com valores de } \mathrm{VO}_{2} \\
25,2 \pm 5,9 \mathrm{ml} / \mathrm{kg} / \mathrm{min}-172 \pm 26 \mathrm{bpm} \mathrm{e} \\
5,4 \pm 1,9 \mathrm{mmol} \text {. }\end{array}$ \\
\hline $\begin{array}{l}\text { Dallmeijer et al., } \\
(2004)\end{array}$ & $\begin{array}{l}\text { Comparar respostas } \\
\text { cardiorrespiratórias em } \\
\text { ergômetro de braço } \\
\text { (para deslocamento } \\
\text { em ruas) e cadeira de } \\
\text { rodas; }\end{array}$ & $\begin{array}{l}9 \text { PLME e } 10 \text { sem } \\
\text { lesão;média de } \\
\text { idade de } 36 \text { anos; }\end{array}$ & $\begin{array}{l}2 \text { séries de } 4 \text { min submáximo } \\
\text { seguidos de } 1 \text { min com } \\
\text { potência crescente até a } \\
\text { exaustão; }\end{array}$ & $\begin{array}{l}\text { Ficou evidenciado a igualdade de } \\
\text { respostas cardiorrespiratórias em ambos } \\
\text { os grupos, mesmo utilizando-se de } \\
\text { diferentes modelos de equipamentos } \\
\text { para a avaliação aeróbia. }\end{array}$ \\
\hline $\begin{array}{l}\text { Hopman et al., } \\
\text { (2004) }\end{array}$ & $\begin{array}{l}\text { Comparar o } \mathrm{VO}_{2} \text { máx } \\
\text { em PLME diferentes } \\
\text { níveis e um grupo não } \\
\text { lesado; }\end{array}$ & $\begin{array}{l}10 \text { controles não } \\
\text { lesados, } 6 \text { tetras e } 6 \\
\text { paraplégicos, média } \\
\text { de idade } 33 \text { anos; }\end{array}$ & $\begin{array}{l}\text { Cicloergômetro de braço } \\
\text { com incremento de carga de } \\
10 \mathrm{~W} / \mathrm{min}\end{array}$ & $\begin{array}{l}\text { Maior } \mathrm{VO}_{2} \text { máx no grupo controle, } \\
\text { seguido do grupo paraplégico e depois } \\
\text { os tetraplégicos. Indicando uma limitação } \\
\text { no } \mathrm{VO}_{2} \text { provavelmente induzido pelo } \\
\text { limitado fornecimento de } \mathrm{O}_{2} \text {. }\end{array}$ \\
\hline
\end{tabular}


... continua

\begin{tabular}{|c|c|c|c|c|}
\hline Autores/ano & Objetivos & Amostra & Materiais e Métodos & Resultados/ Considerações \\
\hline $\begin{array}{l}\text { Knechtle et al., } \\
(2003)\end{array}$ & $\begin{array}{l}\text { Determinar a taxa de } \\
\text { oxidação de gordura } \\
\text { em cicloergômetro de } \\
\text { braço; }\end{array}$ & $\begin{array}{l}10 \text { atletas usuários } \\
\text { de cadeira de } \\
\text { rodas e } 10 \text { ciclistas } \\
\text { treinados; }\end{array}$ & $\begin{array}{l}\text { Avaliação em cicloergômetro } \\
\text { de braço e bicicleta } \\
\text { ergométrica respectivamente; }\end{array}$ & $\begin{array}{l}\text { Os atletas com LME apresentaram } \\
\text { maiores valores de lactato em cada } \\
\text { intensidade quando comparado com } \\
\text { os ciclistas. E os atletas cadeirantes } \\
\text { alcançaram maior oxidação de } \\
\text { gordura no cicloergômetro de braços, } \\
\text { indicando a possibilidade de uso para } \\
\text { treinamento. }\end{array}$ \\
\hline $\begin{array}{l}\text { Paolillo, Paolillo e } \\
\text { Cliquet Jr. (2005) }\end{array}$ & $\begin{array}{l}\text { Avaliar aspectos } \\
\text { cardiorrespiratórios em } \\
\text { para e tetraplégicos; }\end{array}$ & $\begin{array}{l}5 \text { paraplégicos e } 5 \\
\text { tetraplégicos }\end{array}$ & $\begin{array}{l}\text { Avaliações (direta) em } \\
\text { repouso, na estimulação } \\
\text { elétrica neuro-muscular no } \\
\text { quadríceps e na recuperação; }\end{array}$ & $\begin{array}{l}\text { Quanto mais alta a lesão menor } \\
\text { os valores de } \mathrm{VO}_{2} \text { máx. Além de } \\
\text { bradicardia e hipotensão no grupo } \\
\text { tetraplégico. }\end{array}$ \\
\hline Hayes et al., (2005) & $\begin{array}{l}\text { Avaliar o } \mathrm{VO}_{2} \text { máx } \\
\text { em teste incremental } \\
\text { em cicloergômetro de } \\
\text { braço; }\end{array}$ & $\begin{array}{l}13 \text { PLME- } 3 \\
\text { tetraplégicos e uma } \\
\text { mulher com idade } \\
\text { variando de } 30 \text { a } 72 \\
\text { anos; }\end{array}$ & $\begin{array}{l}\text { Avaliados em ergômetro de } \\
\text { braço com analisador } \mathrm{K} 4 \text {; }\end{array}$ & $\begin{array}{l}\text { Média do } \mathrm{VO}_{2} \text { máx foi de } 16,36 \mathrm{ml} / \mathrm{kg} / \\
\text { min. Resultados dentro da média desta } \\
\text { população, mas com grande amplitude } \\
\text { de resultados. }\end{array}$ \\
\hline $\begin{array}{l}\text { Vanlandewijck et } \\
\text { al., (2006) }\end{array}$ & $\begin{array}{l}\text { Avaliar o } \mathrm{VO}_{2} \text { máx } \\
\text { em teste de quadra } \\
\text { e variações de } \\
\text { ambiente/cambagens }\end{array}$ & 7 paraplégicos; & $\begin{array}{l}\text { Análise de gases direta } \\
\text {-portátil (K4); }\end{array}$ & $\begin{array}{l}\text { Valores médio de } \mathrm{VO}_{2} \text { pico } 29,07 \mathrm{ml} / \\
\mathrm{kg} / \mathrm{min} \text { e } \mathrm{FC} \text { máx de } 181 \mathrm{bpm} .\end{array}$ \\
\hline $\begin{array}{l}\text { Goosey-Tolfrey e } \\
\text { Tolfrey (2008) }\end{array}$ & $\begin{array}{l}\text { Correlacionar teste de } \\
\text { quadra com teste em } \\
\text { ergômetro de braço; }\end{array}$ & $\begin{array}{l}24 \text { jogadores } \\
\text { de basquete em } \\
\text { cadeira de rodas } \\
\text {-LME; }\end{array}$ & $\begin{array}{l}\text { Teste de quadra com } \\
\text { incremento de carga e } \\
\text { ergoespirometria direta; }\end{array}$ & $\begin{array}{l}\mathrm{VO}_{2} \text { pico direto média de } 2,66 \mathrm{l} / \mathrm{min} \text { e } \\
\text { Fc máx de } 188 \text { bpm; Teste de quadra } \\
\text { Fc máx de } 186 \text { e distância de } 2056 \mathrm{mts} \text {; } \\
\text { Correlação entre o teste de quadra e o } \\
\text { teste no ergômetro. }\end{array}$ \\
\hline $\begin{array}{l}\text { Leicht, Bischop e } \\
\text { Goosey-Tolfrey } \\
\text { (2011) }\end{array}$ & $\begin{array}{l}\text { Verificar as respostas } \\
\text { em exercício } \\
\text { submáximo }(40 \text { a } \\
80 \% \text { do } \mathrm{VO}_{2} \text { pico; }\end{array}$ & $\begin{array}{l}25 \text { atletas } \\
\text { cadeirantes } \\
\text { (8-tetras; } 9 \text { paras } \\
\text { e } 8 \text { não lesados } \\
\text { medulares) }\end{array}$ & $\begin{array}{l}\text { Análise direta do } \mathrm{VO}_{2} \text { pico, } \\
\text { lactato e Fc máx; }\end{array}$ & $\begin{array}{l}\text { Os tetraplégicos apresentaram os } \\
\text { menores valores }(24,5 \mathrm{ml} / \mathrm{kg} / \mathrm{min}) \\
\text { indicando que a lesão e a altura da } \\
\text { lesão influenciam nas respostas da } \\
\text { potência aeróbia. Sugestão de uso da } \\
\text { FC como prescrição para tetras atletas. }\end{array}$ \\
\hline $\begin{array}{l}\text { Morgulec- } \\
\text { Adamowics et al., } \\
\text { (2011) }\end{array}$ & $\begin{array}{l}\text { Examinar aspectos } \\
\text { aeróbios, anaeróbios } \\
\text { e de habilidades do } \\
\text { RCR com as diferentes } \\
\text { classificações } \\
\text { funcionais; }\end{array}$ & $\begin{array}{l}30 \text { atletas de } \\
\text { RCR da Polônia } \\
\text { subdivididos pela } \\
\text { classificação do } \\
\text { RCR; }\end{array}$ & $\begin{array}{l}\text { Exercício aeróbio máximo } \\
\text { em esteira para cadeira de } \\
\text { rodas com incrementos até } \\
\text { a exaustão; Wingate test em } \\
\text { ergômetro de braço; }\end{array}$ & $\begin{array}{l}\mathrm{VO}_{2} \text { pico } 21,1 \pm 6,3 \text { a } 30,2 \pm 7,2 \mathrm{ml} / \mathrm{kg} \text { / } \\
\text { min; Potência Pico } 83 \pm 35 \text { a } 226 \pm 28 \mathrm{~W} \text {; } \\
\text { Estes resultados demonstraram que a } \\
\text { classificação funcional não interferiu nas } \\
\text { respostas das variáveis avaliadas para } \\
\text { classes intermediárias do jogo. }\end{array}$ \\
\hline Lovell et al. (2012) & $\begin{array}{l}\text { Comparar } \\
\text { as respostas } \\
\text { cardiorrespiratórias } \\
\text { e de eficiencia } \\
\text { mecânica em ciclistas } \\
\text { treinados e não } \\
\text { treinados com LME }\end{array}$ & $\begin{array}{l}20 \text { sujeios com } \\
\text { LME. } 2 \text { grupos: } \\
\text { Atletas e não } \\
\text { atletas }\end{array}$ & $\begin{array}{l}\text { Teste aeróbio submáximo no } \\
\text { ergômetro de braço. Dois } \\
\text { estágios de } 4 \text { minutos por } 1 \\
\text { de intervalo }\end{array}$ & $\begin{array}{l}\text { Grupo atletas apresentou } \mathrm{VO}_{2 \text { pico }} \\
\text { superior }(40.4 \pm 5.5 \mathrm{ml} / \mathrm{kg} / \mathrm{min}) \text { em } \\
\text { comparação com o grupo de não } \\
\text { atletas }(21.23 \pm 4.7 \text { L.min- } 1) \text {. }\end{array}$ \\
\hline
\end{tabular}

Quadro 1 - Sumário dos estudos de pessoas com lesão da medula espinhal e aspectos relacionados a potência aeróbia.

\section{DISCUSSÃO}

A partir da revisão realizada, observa-se uma grande variação de modelos, testes e maneiras de avaliar componentes fisiológicos relacionados aos sistemas cardiorrespiratórios e circulatórios. Esta gama de métodos parece estar relacionada à heterogeneidade da população/amostra, pois as lesões apresentam uma variação muito 
grande de acometimento e diversificações na sua incidência sobre a coluna vertebral. Consequentemente, os resultados, por mais similares que sejam em alguns aspectos, apresentam valores discretos de proximidade, revelando uma necessidade de padronização para estes procedimentos neste grupo amostral. No entanto, essa variação não desmerece nenhum dos trabalhos realizados, ao contrário, serve de base para próximas pesquisas que se alavancam em cada grupo de estudo, sejam elas realizadas no Brasil ou em outra parte do mundo. Outro aspecto que fica evidente nos estudos aqui revisados com pessoas com lesão da medula espinhal é a interferência da altura da lesão nos resultados de variáveis aeróbias - quanto mais baixa a lesão (paraplegia), melhores são as condições aeróbias, em contrapartida, quanto mais alta a lesão (tetraplegia), piores são os resultados das variáveis aeróbias.

Fica clara, igualmente, a importância de utilização de recursos tecnológicos neste campo de pesquisa, pois, com tais recursos, obtém-se menor incidência de erros, proporcionando resultados mais sólidos a serem apresentados perante as avaliações criteriosas de publicações internacionais.

A minimização do erro de medida é fundamental frente a grande heterogeneidade amostral observada nos estudos da área. Compreende-se, também, que estes estudos podem servir como ponto de partida para a elaboração de testes indiretos, que possam ser utilizados no dia a dia de trabalho de pessoas com lesão da medula espinhal.

Os dados levantados neste trabalho permitem a guisa de conclusão estabelecer algumas considerações sobre o tema em questão. Os métodos utilizados nos estudos analisados evidenciam desde testes indiretos com ampla aplicabilidade até técnicas laboratoriais com equipamentos de elevado custo e grande precisão. Já em relação as variáveis analisadas o consumo máximo de oxigênio, valores de potência aeróbia e cargas máximas em watts alcançadas nos testes foram destacados nesta revisão. A heterogeneidade da amostra é um fator que vai influenciar diretamente os parâmetros cardiovasculares bem como os parâmetros relativos à avaliação. É necessária a investigação de protocolos acessíveis de campo que possam ser aplicáveis à sedentários, pois os protocolos existentes são mais voltados ao público fisicamente ativo. Faz-se necessária a consolidação de protocolos laboratoriais e para tanto uma opção interessante e acessível é o ergômetro de membros superiores. Sanar estas questões é imperativo para a proposição de normas referenciais, as quais são fundamentais para a prescrição de exercícios nesta população.

\section{REFERÊNCIAS}

1. Myers J,Lee M, Kiratli J. Cardiovascular disease in spinal cord injury: an overview of prevalence, risk, evaluation, and management. Am J Phys Med Rehabil. 2007; 86:142-52.

2. Kemi OJ, Wisloff U. High-intensity aerobic exercise training improves the heart in health and disease. J Cardiopulm Rehabil Prev. 2010; 30:2-11.

3. Gulati M, Pandey DK, Arnsdorf MF, Lauderdale DS, Thisted RA, Wicklund RH. et al. Exercise capacity and the risk of death in women. The St James women take heart project. Circulation. 2003; 108:1554-9.

4. Dencker M, Thorsson O, Karlsson MK, Linde'N C, Wollmer P, Andersen LB. Maximal oxygen uptake versus maximal power output in children. J Sports Sci. 2008; 26: 1397-1402.

5. Paolillo FR, Paolillo AR, Cliquet-JR A. Respostas cardio-respiratórias em pacientes com traumatismo raquimedular. Acta Orto Bras. 2005; 13 (3).

6. Lavis TD, Scelza WM, Bockenek WL. Cardiovascular health and fitness in person with spinal cord injury. Phys Med Rehabil Clin N Am. 2007;18:317-331.

7. Stevens SL, Caputo JL, Fuller DK, Morgan DW. Physical Activity and Quality of Life in Adults With Spinal Cord injury. J Spinal Cord Med. 2008; 31(4):373-8. 
8. Noronha DP, Ferreira SMSP. Revisões de literatura. In: Campello BS, Condón BV, Kremer JM. (orgs.) Fontes de informação para pesquisadores e profissionais. Belo Horizonte: UFMG, 2000.

9. Haisma JA, Woude LHV, Stam HJ, Bergen MP, Sluis T, Bussmann JBJ. Physical capacity in wheelchair-dependent persons with a spinal cord injury: a critical review of the literature. Spinal cord. 2006; 44: 642-652.

10. Theisen D, Vanlandewijck Y. Cardiovascular responses and thermoregulation in individuals with spinal cord injury. Eur Bul Adapted Phys Activity. 2002; 1: (1).

11. Teasell RW, Arnold JM, Krassioukov A, Delaney GA. Cardiovascular consequences of loss of supraspinal control of the sympathetic nervous system after spinal cord injury. Arch Phys Med Rehabil. 2000; 81(4):506-16.

12. Eriksson P, Lofstrom L, Ekblom B. Aerobic power during maximal exercise in untrained and well-trained persons with quadriplegia and paraplegia. Scand J Rehabil Med. 1988; 20:141-7.

13. Lovell D, Shields D, Beck B, Cuneo R, McLellan C. The aerobic performance of trained and untrained handcyclists with spinal cord injury. Eur J Appl Physiol. 2012; 112:3431-3437.

14. Bernard PL, Mercier J, Varray A, Prefaut C. Influence of lesion level on the cardioventilatory adaptations in paraplegic wheelchair athletes during muscular exercise. Spinal Cord. 2000; 38:16-25.

15. Knechtle B, Kopfli W.Treadmill exercise testing with increasing inclination as exercise protocol for wheelchair athletes. Spinal Cord. 2001; 39: 633-636.

16. Jacobs PL, Nash MS, Rusinowski JW. Circuit training provides cardiorespiratory and strength benefits in persons with paraplegia. Med Sci Sports Exerc. 2001;33: 711-717.

17. Bougenot MP, Tordi N, Betik AC, Martin X, Le Foll D, Parratte B, Lonsdorfer J, Rouillon JD. Effects of wheelchair ergometer training program me on spinal cord-injured persons. Spinal Cord. 2003; 41: 451-456.

18. Vanderthommen M, Francaux M, Colinet C, Lehance C, Lhermerout C, Crielaard JM, Theisen D. A multistage field test of wheelchair users for evaluation of fitness and prediction of peak oxygen consumption. J Rehabil Res Dev. 2002; 39(6):685-92.

19. Janssen TW, Dallmeijer AJ, Veeger DJ, Van Der Woude LH. Normative values and determinants of physical capacity in individuals with spinal cord injury. J Rehabil Res Dev. 2002; 39: 29-39.

20. Dallmeijer AJ, Zentgraaff ID, Zijp NI, Van Der Woude LH. Submaximal physical strain and peak performance in hand cycling versus hand rim wheelchair propulsion. Spinal Cord. 2004; 42: 91-98.

21. Hopman MT, Houtman S, Groothuis JT, Folgering HT. The effect of varied fractional inspired oxygen on arm exercise performance in spinal cord injury and able-bodied persons. Arch Phys Med Rehabil. 2004; 85: 319-323.

22. Knechtle B, Muller G, Willmann F, Eser P, Knecht H. Comparison of fat oxidation in arm cranking in spinal cord-injured people versus ergometry in cyclists. Eur J Appl Physiol. 2003; 90: 614-619.

23. Hayes AM, Myers JN, Ho M, Lee MY, Perkash I, Kiratli BJ. Heart rate as a predictor of energy expenditure in people with spinal cord injury. J Rehabil Res Dev. 2005; 42(5):617-24.

24. Vanlandewijck Y, Vliet PV, Verellen J, Theisen D. Determinants of shuttle run performance in the prediction of peak VO2 in wheelchair users. Disabil Rehabil. 2006; 28 (20): 1259-1266.

25. Goosey-Tolfrey VL, Tolfrey K. The multi-stage fitness test as a predictor of endurance fitness in wheelchair athletes. J Sports Sci. 2008; 26(5):511-7.

26. Goosey-Tolfrey VL. Physiological profiles of elite wheelchair basketball players in preparation for the 2000 Paralympics games. Adapt Phys Activ Q.2005; 22:57-66, 2005.

27. Leicht CA, Bishop NC, Goosey-Tolfrey VL. Submaximal exercise responses in tetraplegic, paraplegic and non spinal cord injured elite wheelchair athletes. Scan J Med Sci Sports 2011; 23.

28. Morgulec-Adamowicz N, Kosmol A, Molik B, Yilla A, Laskin J. Aerobic, anaerobic, and skill performance with regard to classification in wheelchair rugby athletes. Res Q Exerc Sport. 2011; 82(1):61-9, 2011.

$$
\begin{array}{r}
\text { Endereço para Correspondência } \\
\text { Lucinar J. Forner. Flores } \\
\text { Av. Arnaldo Busatto, } 210 \text { apto 102- } \\
\text { Centro- Santa Helena-PR. } \\
\text { CEP } 85892-000 \\
\text { Telefone celular: (45) 9919-5226 Tele- } \\
\text { fone comercial: (45) 3284-7855 } \\
\text { E-mail: lucinarflores@yahoo.com.br }
\end{array}
$$

Recebido 03/04/2012

Revisado 24/04/2012 $13 / 09 / 2012$ $25 / 10 / 2012$

Aprovado 31/10/2012 